\title{
When is re-irradiation in head and neck squamous cell carcinoma not indicated?
}

\author{
Primož Strojan • Jonathan J. Beitler • Carl E. Silver • \\ William M. Mendenhall • Ashok R. Shaha - Alessandra Rinaldo • \\ Robert P. Takes • Alfio Ferlito
}

Received: 14 October 2013/Accepted: 28 October 2013/Published online: 9 November 2013

(c) Springer-Verlag Berlin Heidelberg 2013

At least as reflected by the literature, the popularity of reirradiation (rRT) for head and neck squamous cell carcinoma (HNSCC) has increased greatly in the last few years [1]. Guidelines are emerging [2, 3]. There are four reasons, two of them "old" and two of them influenced by recent events. First, despite continuous refinement of treatment strategies, HNSCC remains a locoregional disease with a failure rate above the clavicles in the range of $50 \%$ [4]. Second, even in the human papillomavirus (HPV) era, these patients are subject to an increased risk of second HNSCC [5]. The third reason is that with better imaging, we can detect more local and regional recurrences when they are smaller and more amenable to either surgery or radiotherapy (RT). Finally, intensity modulated radiation therapy (IMRT), volumetric modulated arc therapy (VMAT), stereotactic body radiosurgery (SBRS), tomotherapy, improved brachytherapy and protons technology

The paper was written by members of the International Head and Neck Scientific Group (http://www.IHNSG.com).

P. Strojan

Department of Radiation Oncology, Institute of Oncology,

Ljubljana, Slovenia

\section{J. J. Beitler}

Department of Radiation Oncology, Winship Cancer Institute,

Emory University, Atlanta, GA, USA

C. E. Silver

Departments of Surgery and Otolaryngology-Head and Neck Surgery, Montefiore Medical Center, Albert Einstein College of Medicine, Bronx, NY, USA

W. M. Mendenhall

Department of Radiation Oncology, College of Medicine,

University of Florida, Gainesville, FL, USA allow us to deliver rRT with increased precision and hence, better patient tolerability $[6,7]$.

Decisions about therapy for recurrence are best undertaken in a multidisciplinary setting. At this moment, surgical salvage still remains the gold standard of care for these patients. The literature of rRT is unclear because it is dominated by low-level evidence from many retrospective studies with prospective data being sparse [3]. To ensure a maximum benefit from rRT, it is valuable to look at the problem of rRT from the opposite perspective. If we agree on "when is rRT not indicated?", it would lend clarity to discussions at tumor boards. There are several principles that should be respected when considering rRT.

First, a curative resection should be the first consideration. Although not confirmed in a prospective and randomized fashion, one may infer that complete removal of macroscopic and microscopic tumor would optimize the chance for local control. This assumption is supported by a negative correlation between the tumor bulk left behind after salvage surgery and the survival outcome found in some studies [8]. Another argument could be raised in terms

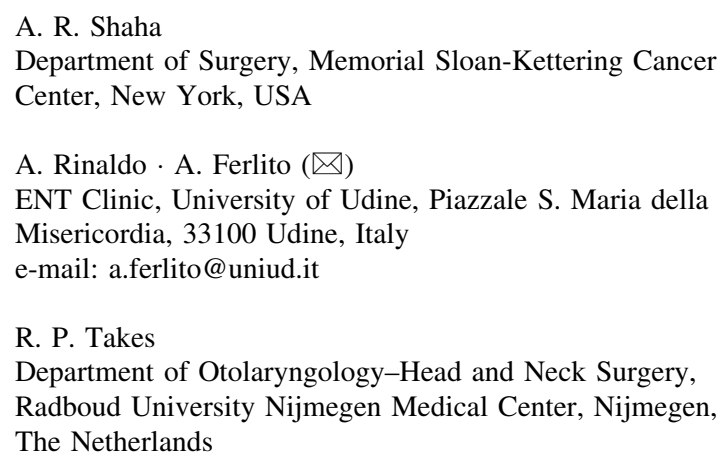


of the difference in survival rates between the operated and re-irradiated only patients, which cannot be explained by a simple bias related to the volume of the treated disease. Moreover, previous chemoradiation was found to adversely influence survival of patients undergoing rRT, presumably due to the acquired therapeutic resistance of surviving clonogens. Surgical removal of gross tumor mass before rRT circumvents this problem [9]. Finally, a meta-analysis of 32 surgical studies with 1,080 patients demonstrated a survival rate of $39 \%$ at 5 years in patients treated by salvage surgery, compared with a 20-30\% overall survival rate at 2 years after $\mathrm{rRT}$ alone [3, 10].

Second, patients with significant toxicity from previous RT are not suitable candidates for rRT.

Third, comorbidity must be assessed (and this holds true for all modalities). For an objective assessment of the patients' functional status, the use of standardized measures (e.g., the Charlson Comorbidity Index and the Adult Comorbidity Evaluation-27 [ACE-27] grading) is highly appropriate. With regard to the importance of comorbidity and pre-existing organ dysfunction in an rRT setting, the most informative results were obtained from the study of Tanvetyanon et al. [8] of a retrospective series of 103 patients undergoing rRT with different techniques between 1998 and 2008, of whom 46 (45\%) had salvage surgery before rRT. The Charlson Index and ACE-27 grading were used to assess comorbidity. Organ dysfunction was defined as feeding tube dependency, functioning tracheostomy or a soft-tissue defect. In a multivariate model, both comorbidity and organ dysfunction were identified as significant negative predictors of overall survival. No long-term survivors were observed among patients with both comorbidity and organ dysfunction; median survival in this group was 5.5 months as compared to 59.6 months for those without either of these predictors.

Fourth, as a general rule, rRT should not be offered when the time interval from the previous RT is $<6$ months. This time limit was set arbitrarily and was based on empirical rather than scientific grounds, as the majority of studies used the cut off point of 6 months as one of the criteria for the recruitment of patients for rRT [3]. In the prospective RTOG 9610 study and two out of three largest retrospective series (each with $>100$ patients included), the interval since last RT positively correlated with the survival outcome, with the time cut off point usually identified at 36 months [8, 9, 11, 12]. It appears that with regard to re-treatment interval, the premise should be "longer is better", although no impact of delay from first irradiation and opposite observations has been reported in the literature [12-14]. This rule probably does not apply if the previous radiation missed the area of recurrence.

Finally, there is the question of the rRT dose that can be delivered in particular patients. Due to the already existing dose burden to different tissues caused during the first course of RT, the balance between two objectives, effectiveness and safety, is much more delicate in the rRT setting. The first intention-to destroy the tumor-requires a high irradiation dose, preferably of $60 \mathrm{~Gy}$ or more. The other intention - not to do harm to the patient - requires the opposite, i.e., minimization of the dose to the tissues surrounding the treated target. Owing to smaller rRT target volumes, which are usually limited to gross tumor and without the intention to electively irradiate adjacent regions, acute toxicity during a repeated course of RT is usually comparable to that produced by the first irradiation course, but long-term morbidity after rRT can be substantial. Despite the capacity of some late-reacting tissues to recover from occult radiation injury (e.g., spinal cord) as well as a significant improvement in isodose shaping and distribution achieved using modern RT technology, the reported incidence of late toxicity is still a highly pertinent issue [3]. For example, in the previously mentioned study of Tanvetyanon et al. [8], the reported incidence of late toxicities of grade 3 or higher was $47.5 \%$, whereas in the series of Choe et al. [9], $68 \%$ of patients had a gastrostomy tube in place at last follow-up (median follow-up time of alive patients: 53 months); the corresponding figure reported in the RTOG 9610 study was $70 \%$ [11]. It appears, however, that the implementation of modern RT techniques could effectively reduce the incidence of late toxicities [15]. Pre-existing tissue changes produced by the original course of RT should be taken into consideration when making a case for rRT rather than only considering a cumulative dose distribution of the previous and actual RT courses. Obviously, the existing window that allows burdening the pre-irradiated late-reacting tissues with an additional dose is usually narrow, and when it prevents the delivery of a sufficiently high irradiation dose to the target, the purpose of rRT, in terms of benefits for the patient, should be seriously questioned.

How best quantify the assessment of patients' suitability for rRT? One option is to use nomograms, which incorporate different patient-, disease- and treatment-related factors with a significant impact on the treatment outcome and survival. The first of such nomograms was created by Tanvetyanon et al. [8] based on the analysis of their own experience with rRT. The observed end-point was the probability of death within 24 months after rRT, and only those variables that were found significant in the multivariate analysis of overall survival were included in the statistical model, taking into account their contribution to the accuracy of prediction: comorbidity (assessed by the Charlson Index or ACE-27), pre-existing organ dysfunction, isolated neck recurrence, tumor volume, and delay after previous RT course. Using this nomogram, the authors observed a good agreement between the observed 
and predicted outcomes, which was recently confirmed by Shikama et al. [16] who repeated the analysis using data from their own series of patients. Based on the tested nomogram, curative-intent rRT is strongly recommended for patients without comorbidity and organ dysfunction and with isolated neck recurrence, whereas palliative-intent rRT is indicated for patients with adverse prognostic factors only in the case of symptomatic or gross disease [8]. Patients who develop an isolated local or regional recurrence due to a marginal miss or underdosage are probably more likely to benefit from rRT to a greater extent than those who experience a recurrence well within the high dose volume, particularly if it is both local and regional [17]. Whether or not the use of a nomogram is the best method to clarify the issue of patients' selection for rRT should be tested in a prospective fashion, also allowing the collection of other pertinent information on organ dysfunction (e.g., fibrosis, xerostomia, trismus, chronic pain) and treatment (e.g., degree of overlap between previous and present irradiation fields, exact target volume), which is usually not available in retrospective analyses.

The management of locoregional recurrence in HNSCC is a very difficult and complex problem. The decisions are best made in a truly multidisciplinary fashion with direct input from all specialties, including imaging and pathology specialists. The board's first responsibility is to decide whether the tumor can be curatively resected. If the tumor cannot be resected for technical or functional reasons, then alternate choices must be considered. rRT does engender serious complications which need to be properly weighed against the benefits. However, we need to recognize that these patients have no other effective definitive treatment available, and we must accept some inevitable radiation related complications such as dysphagia, ulceration and neurological damage. The patient's understanding of these complex issues and acceptance of such complications is critical.

\section{References}

1. Nieder C, Andratschke NH, Grosu AL (2013) Increasing frequency of reirradiation studies in radiation oncology: systematic review of highly cited articles. Am J Cancer Res 3:152-158

2. McDonald MW, Lawson J, Garg MK et al (2011) ACR appropriateness criteria retreatment of recurrent head and neck cancer after prior definitive radiation expert panel on radiation oncology-head and neck cancer. Int $J$ Radiat Oncol Biol Phys 80:1292-1298

3. Strojan P, Corry J, Eisbruch A et al (2013) Recurrent and second primary squamous cell carcinoma of the head and neck: when and how to re-irradiate. Head Neck (in press)

4. Blanchard P, Baujat B, Holostenco V et al (2011) Meta-analysis of chemotherapy in head and neck cancer (MACH-NC): a comprehensive analysis by tumour site. Radiother Oncol 100:33-40

5. Erkal HS, Mendenhall WM, Amdur RJ, Villaret DB, Stringer SP (2001) Synchronous and metachronous squamous cell carcinomas of the head and neck mucosal sites. J Clin Oncol 19:1358-1362

6. Malicki J (2012) The importance of accurate treatment planning, delivery, and dose verification. Rep Pract Oncol Radiother 17:63-65

7. Scala LM, Hu K, Urken ML et al (2013) Intraoperative highdose-rate radiotherapy in the management of locoregionally recurrent head and neck cancer. Head Neck 35:485-492

8. Tanvetyanon T, Padhya T, McCaffrey J et al (2009) Prognostic factors for survival after salvage reirradiation of head and neck cancer. J Clin Oncol 27:1983-1991

9. Choe KS, Haraf DJ, Solanki A et al (2011) Prior chemoradiotherapy adversely impacts outcomes of recurrent and second primary head and neck cancer treated with concurrent chemotherapy and reirradiation. Cancer 117:4671-4678

10. Goodwin WJ Jr (2000) Salvage surgery for patients with recurrent squamous cell carcinoma of the upper aerodigestive tract: when do the ends justify the means? Laryngoscope 110(Suppl 93): $1-18$

11. Spencer SA, Harris J, Wheeler RH et al (2008) Final report of RTOG 9610, a multi-institutional trial of reirradiation and chemotherapy for unresectable recurrent squamous cell carcinoma of the head and neck. Head Neck 30:281-288

12. De Crevoisier R, Bourhis J, Domenge C et al (1998) Full-dose reirradiation for unresectable head and neck carcinoma: experience at the Gustave-Roussy Institute in a series of 169 patients. J Clin Oncol 16:3556-3562

13. Langer CJ, Harris J, Horwitz EM et al (2007) Phase II study of low-dose paclitaxel and cisplatin in combination with split-course concomitant twice-daily reirradiation in recurrent squamous cell carcinoma of the head and neck: results of Radiation Therapy Oncology Group Protocol 9911. J Clin Oncol 25:4800-4805

14. Milanović D, Jeremić B, Grosu AL, Rücker G, Henke M (2013) Reirradiation plus EGFR inhibition in locally recurrent and unresectable head and neck cancer: final results from a single institution. Strahlenther Oncol 189:842-848

15. Kharofa J, Choong N, Wang D et al (2012) Continuous-course reirradiation with concurrent carboplatin and paclitaxel for locally recurrent, nonmetastatic squamous cell carcinoma of the head-and-neck. Int J Radiat Oncol Biol Phys 83:690-695

16. Shikama N, Kumazaki Y, Tsukamoto N et al (2013) Validation of nomogram-based prediction of survival probability after salvage re-irradiation of head and neck cancer. Jpn J Clin Oncol 43:154-160

17. Mendenhall WM, Mendenhall CM, Malyapa RS, Palta JR, Mendenhall NP (2008) Re-irradiation of head and neck carcinoma. Am J Clin Oncol 31:393-398 